\title{
Paracrine Effects Of Adipose-Derived Stromal/Stem Cells And Stromal Vascular Fraction In An In Vitro Fibrogenesis Model Of Human Vocal Fold Scarring
}

\section{Mélanie Velier}

Assistance Publique Hopitaux de Marseille https://orcid.org/0000-0002-1121-8270

\section{Alexia MATTEI}

Assistance Publique Hopitaux de Marseille

\section{Stéphanie SIMONCINI}

Aix-Marseille Universite

Jérémy MAGALON

Assistance Publique Hopitaux de Marseille

Laurent GIRAUDO

Assistance Publique Hopitaux de Marseille

\section{Laurent ARNAUD}

Assistance Publique Hopitaux de Marseille

\section{Antoine GIOVANNI}

Assistance Publique Hopitaux de Marseille

Francoise DIGNAT GEORGE

Aix-Marseille Universite

Florence SABATIER ( $\sim$ florence.sabatier@ap-hm.fr )

Markus GUGATSCHKA

Medizinische Universitat Graz

\section{Tanja GROSSMANN}

Medizinische Universitat Graz

\section{Short report}

Keywords: adipose derived stromal cells, fibrosis, macromolecular crowding, vocal fold scarring

Posted Date: April 2nd, 2020

DOI: https://doi.org/10.21203/rs.3.rs-20745/v1

License: (c) (i) This work is licensed under a Creative Commons Attribution 4.0 International License.

Read Full License 


\section{Abstract}

Background: Vocal folds (VF) scarring leads to severe dysphonia which negatively impacts daily life of patients. Current therapeutic options are limited due in large part to the high complexity of the microstructure of the VF. Innovative therapies derived from adipose tissue such as stromal vascular fraction (SVF) or adipose derived stromal/ stem cells (ASC) are currently being evaluated in this indication and paracrine anti-fibrotic effects are considered as predominant mechanisms.

Methods: The paracrine anti-fibrotic effects of SVF and ASC from healthy donors were tested in an innovative in vitro fibrogenesis model employing human VF fiboblasts (hVFF) and the principles of macromolecular crowding (MMC). Biosynthesis of collogen and alpha-smooth-muscle actin (aSMA) expression in hVFF were quantified after five days of indirect coculture with ASC or SVF using silver stain, western blot and RT-qPCR analysis.

Results: Fibrogenesis was promoted by addition of transforming growth factor beta 1 (TGF $\beta 1$ ) combined with MMC characterized by an enhanced deposition of fibrillar collagens and the acquisition of a myofibroblast phenotype (overexpression of aSMA). Adipose-derived therapies led to a reduction in the aSMA expression and the collagen content was lower in hVFF co-cultivated with SVF.

Discussion: ASC and SVF promoted significant prevention of fibrosis in an in vitro fibrogenesis model through paracrine mechanisms, supporting further development of adipose-derived cellular therapies in VF scarring.

\section{Introduction}

Vocal fold (VF) scar is one of the most challenging benign laryngeal pathologies [1]. Depending on severity and extent, it can result in a range of causes both physical and psychological disability, especially for persons with a high vocal demand, such as teachers, singers or call center agents.

VF microstructure is complex [2], particularly due to its foliated organization allowing optimal vibration. The proportions and the organization of the extracellular matrix (ECM) components largely determine the mechanical properties of the VF. VF scarring is most often the result of laryngeal microsurgery, but it may also be found congenitally (sulcus vocalis) oracquired following a trauma or chronic inflammatory phenomena. Thus, the normal microstructure is replaced by fibrous tissue, with an excessive and disorganized ECM inducing vibration disorder [1]. Compared to normal fibroblasts, VF scar tissue fibroblasts produce a larger amount of collagen, proliferate more rapidly and overexpress a-smoothmuscle actin (aSMA), also known as actin alpha 2 (ACTA2), a myofibroblast differentiation marker [3, 4].

Despite recent advances (medialization, scar resection, microflap, injection of hyaluronic acid, etc.) [5-8], current therapies are often disappointing since they do not restore a normal ECM distribution. Other more experimental treatments such as administration of basic Fibroblast Growth Factor (bFGF) $[9,10]$ or Hepatocyte Growth Factor (HGF) [11-14] have shown encouraging effects in vitro or in vivo on animals. 
However, only one phase I/II clinical trial assessed the safety and effectiveness of intracordal injection of a recombinant human HGF in patients with vocal fold scar or sulcus [15]. The results of this first-in-man clinical trial indicated no serious adverse events and encouraging clinical results. Thus, the identification of innovative strategies able to improve the mechanical vibrational properties of VF remains an important clinical challenge.

Mesenchymal stromal cells (MSC), which were first described in bone marrow to support hematopoiesis [16], are multipotent stem cells that can be isolated from a variety of tissues but adipose tissue remains a very attractive source due to its abundance and accessibility. Autologous adipose-derived Stromal Vascular Fraction (SVF) is a heterogeneous population of cells obtained in a few hours after enzymatic digestion of adipose tissue that does not require an expansion step. SVF is also an advantageous source of adipose derived stromal cells (ASC) because of its fluidity and ease to inject. Besides, SVF brings the synergistic effect of its various cellular subpopulations, including ASC but also endothelial progenitor cells combining vasculogenic, anti-fibrotic and anti-inflammatory properties. The exact mechanism of action of ASC and SVF are not fully elucidated but it is now admitted that the major effect rests on paracrine activity through a large type of secreted molecules [17-19].

To facilitate research on this field, Graupp et al. described an in vitro fibrogenesis model using human vocal fold fibroblasts (hVFF) and the principles of macromolecular crowding (MMC) [20]. MMC promotes fibrogenesis after the addition of transforming growth factor-beta 1 (TGF- $\beta 1$ ) to a medium containing inert macromolecules. The aim of this experimental study was to assess the in vitro paracrine potential of SVF cells and ASC on scarred hVFF in this innovative model.

\section{Materials And Methods}

\section{Donors}

ASC and SVF cells from healthy donors $(n=4)$ were obtained from adipose tissue surgical residues following liposuction for aesthetic purposes. All patients provided informed consent for the scientific use of surgical residues.

\section{Cell culture}

Immortalized human vocal fold fibroblasts (hVFF) were obtained from the lab of Prof. Susan Thibeault (University of Wisconsin, Madison, USA) [21]. Dulbecco's modified Eagle's medium (DMEM) (Life Technologies, Waltham, Massachusetts) supplemented with $10 \%$ fetal calf serum (FCS; Sigma-Aldrich, Vienna, Austria) and $100 \mu \mathrm{g} / \mathrm{mL}$ Normocin (Invivogen, San Diego, California, USA) was used as standard medium (SM). Adipose tissue collection was conducted under conscious sedation with local anesthesia. SVF was obtained in a Cell Therapy Unit using the automated processing Celution 800/CRS system (Cytori Therapeutics Inc., San Diego, California, USA) as previously described [22]. Collected lipoaspirate was washed and enzymatically digested to obtain SVF in accordance with the European Regulations and Good Manufacturing Practices (GMP) for 'Advanced Therapy Medicinal Products'. To isolate the ASC, 
SVF was then plated into a T75 cell culture flask in a culture medium consisting of DMEM supplemented with 50\% Ham's F-12 Nutrient Mix (Thermo Scientific, Waltham, Massachusetts, USA ), 10\% FCS, GlutaMAX (100X, Thermo Scientific), Gentamicin and Penicillin G (both Panpharma, Luitré, France), Fungizone (Bristol-Meyers Squibb, New York, NY, USA). Adherent cells were grown under standard cell culture conditions ( $5 \% \mathrm{CO}_{2}, 37^{\circ} \mathrm{C}$, humidified), detached with Trypsin-EDTA and passed to extend the culture. For co-culture experiments, hVFF from passages 13-20 were seeded into cell culture plates $\left(15.000\right.$ cells $\left./ \mathrm{cm}^{2}\right)$. The following day, SM was switched to FCS-free SM for another $24 \mathrm{~h}$. Cells were then allocated to different treatment groups. For the control group DMEM enriched with $0.5 \% \mathrm{FCS}, 100 \mu \mathrm{g} / \mathrm{mL}$ Normocin and $100 \mathrm{nM}$ of L-ascorbic acid 2-phosphate was used [23]. Myofibroblast differentiation was induced by adding of $5 \mathrm{ng} / \mathrm{mL}$ TGF- $\beta 1$ [24] combined with "macromolecular crowding" (MMC) by adding a mixture of $37.5 \mathrm{mg} / \mathrm{mL} 70 \mathrm{kDa}$ Ficoll (Fc) with $25 \mathrm{mg} / \mathrm{mL} 400 \mathrm{kDa}$ Fc (both Sigma-Aldrich) [25, 26]. To assess possible anti-fibrotic effects, three conditions were tested in co-culture with hVFF: (i) recombinant HGF (50 ng/mL, Sigma-Aldrich), (ii) paracrine effect of thawed SVF and (iii) paracrine effect of thawed ASC from passages 3-5. After 5 days of incubation, samples were further processed.

\section{Reverse Transcription-qPCR (RT-qPCR)}

RNA isolation of hVFF was performed using the QIAZOL Lysis Reagent (Qiagen, Hilden, Germany) and total mRNA was isolated with the miRNeasy Mini Kit (Qiagen) according to the manufacturer's instructions. Purified RNA was eluated in RNAse-free water and concentration was determined using the NanoDrop 2000c spectrophotometer (Thermo Scientific). Reverse transcription (RT), as well as RT quantitative PCR (RT-qPCR), was performed as previously described [27]. Primer sequences are provided in Table 1. Relative quantification of all mRNAs of interest was performed based on the $2^{-\Delta \Delta C}{ }_{T}$ method [28].

\section{Pepsin digestion, sodium dodecylsulphate-polyacrylamide gel electrophoresis (SDS-PAGE) and silver stain}

Supernatants and cell layers from hVFF seeded in 24-well-plates were harvested separately and pepsinized as previously described [29]. Briefly, $50 \mu$ of a pepsin stock solution $(1 \mathrm{mg} / \mathrm{mL}$ dissolved in $1 \mathrm{~N}$ $\mathrm{HCL}$ ) were added to $500 \mu \mathrm{L}$ of supernatants, while a pepsin digestion solution (25\% pepsin stock solution, $0.005 \%$ Triton $\mathrm{X}-100$ in $\mathrm{ddH}_{2} \mathrm{O}$ ) was added to cell layers. All samples were incubated for $2 \mathrm{~h}$ followed by neutralization with $1 \mathrm{~N} \mathrm{NaOH}$.

SDS-PAGE was performed under non-reducing conditions using 3-8\% precast Criterion XT Tris-Acetate gels and XT Tricine running buffer (both Bio-Rad, Hercules, California, USA); electrophoresis was run for $70 \mathrm{~min}$ at $200 \mathrm{~V}$. Vitro-Col, human collagen I solution served as collagen standard ( $0.16 \mu \mathrm{g} / \mathrm{lane})$. Gels were subsequently stained with the SilverQuest ${ }^{\text {TM }}$ Silver Staining kit (Thermo Scientific) according to the manufacturer's protocol. Gel images were acquired using Quantity One software (Bio-Rad) and densitometric analysis of bands was performed using Image Lab Software, Version 5.2.

\section{Western blot}


Proteins were extracted from hVFF cell layers seeded in 6-well-plates and subjected to SDS-PAGE using 4-12\% Criterion XT Bis-Tris Gels (Bio-Rad). SDS-PAGE was run at $200 \mathrm{~V}$ for $60 \mathrm{~min}$, followed by electroblotting of proteins ( $90 \mathrm{~min}$ at $0.5 \mathrm{~A}$ and $4{ }^{\circ} \mathrm{C}$ ) onto Nitrocellulose membranes (Bio-Rad). Immunodetection was carried out in Tris-buffered saline supplemented with $0.1 \%$ Tween-20 (Carl Roth, Karlsruhe, Germany) and $5 \%$ milk. Membranes were incubated overnight at $4{ }^{\circ} \mathrm{C}$ with primary antibodies for detection of ACTA2 (\#A5228, Sigma Aldrich, 1:1000) and glyceraldehyde-3-phosphate dehydrogenase (GAPDH; \#2118C, Cell Signaling, Danvers, Massachusetts, USA, 1:5000). Subsequently, blots were incubated with secondary antibody (Goat Anti-Mouse 1:5000 for ACTA2; Goat Anti-Rabbit 1:5000 for GAPDH, both abcam, Cambridge, UK). Detection was conducted using the SuperSignal West Pico Chemiluminescent Substrate (Thermo Scientific) and the ChemiDoc Touch Imaging System (bio-rad), densitometric analysis was performed using Image Lab Software, Version 5.2.

\section{Results}

\section{Validation of the MMC/TGF- $\beta 1$ model}

We first validated the myofibroblast differentiation induced by TGF- $\beta 1$ combined with MMC (MMC/TGF$\beta 1$ ) through the assessment of ACTA2 and Collagen la1 (COL1A1) contents in hVFF. Results from RTqPCR analysis revealed that mRNA expression of ACTA2 and COL1A1 were significantly up-regulated under MMC/TGF- $\beta 1$ condition ( $p=0.019$ and $p=0.002$ respectively) (Fig. $1 \mathrm{~A}$ and $2 \mathrm{~A}$ ) compared to the negative condition (untreated fibroblasts). Western blot and silver stain analysis performed on hVFF protein lysates were in line with the previous data: we observed a significant elevation of the ACTA2/GADPH ratio $(p=0.024)$ and of fibrillar collagen contents $(p=0.0004)$ in the MMC/TGF- $\beta 1$ condition compared to the untreated condition (Fig. 1B and 1C). Altogether, these data validated the myofibroblast phenotype acquisition of immortalized hVFF after MMC/TGF- $\beta 1$ treatment.

\section{Co-culture}

The expression of the myofibroblast marker ACTA2 and collagen content of hVFF co-cultured with ASC or SVF or treated with HGF for 5 days were assessed. The results revealed a reduction in the mRNA level of ACTA2 on hVFF when they were co-cultured with ASC and SVF ( $p=0.043$ and $p=0.028$ respectively) (Fig. 1A). Western blot analysis confirmed these results highlighting a tendency to reduce the level of ACTA2 in hVFF co-cultured with both ASC and SVF ( $p=0.061$ and $p=0.083$ respectively) (Fig. 1B and 1C).

Figure 2 represents the expression of mRNA of COL1A1 and fibrillar collagen in protein lysates of hVFF. We observed no significant reduction in the COL1A1 mRNA expression (Fig. 2A) in both ASC and SVF coculture conditions whereas silver stain analysis revealed a significant reduction of fibrillar collagen content in hVFF after co-culture with SVF $(p=0.006)$ (Fig. 2B and $2 C)$. Of note, no significant reduction of ACTA2 or collagen levels were obtained when hVFF were treated with HGF (Figs. 1 and 2). 


\section{Discussion}

Vocal fold scarring remains one of the most difficult conditions to treat among benign VF diseases and adipose-derived cell therapies represent hope for these patients. The therapeutic potential of MSC has largely been attributed to paracrine activity [30,31]. In this study, we demonstrated that ASC and SVF promoted significant prevention of fibrosis in an in vitro model through paracrine mechanisms.

Previously, a similar study explored whether ASC have therapeutic potential for treating vocal fold scarring through the effects of HGF on scar fibroblasts in an indirect co-culture model [4]. They showed that co-culture with ASC led to a significant decrease of ACTA2 expression in fibroblasts comforting our data. However, they also demonstrated a reduction in collagen production in the ASC co-culture conditions whereas, in our study, we did not observe significant effect of ASC on the fibrillar collagen content.

Compared with ASC, SVF can be real time obtained in a sufficient quantity without in vitro culture, reducing drastically the delay of preparation. Yasuda et al. reported that injection of SVF had a protective effect in an acute kidney injury in vivo model by secreting renoprotective molecules, such as HGF and VEGF [32]. We can hypothesize that in the present study, SVF and ASC reduced the ACTA2 and collagen expression in hVFF partly by secreting those growth factors transferred through the co-culture system. Only conditions with SVF showed a reduction in both ACTA2 and collagen contents which might be attributed to the higher secretion of soluble mediators by SVF. In line with this, the secretome of ASC and SVF has been compared recently and revealed that SVF released a greater variety of cytokines or soluble protein at a significantly higher amounts as compared with ASC [33].

Several in vivo studies on animals reported significant improvements in scarred vocal folds after injection of ASC (immediately after injury or at a longer interval) in both histological and macroscopic aspects of the vocal folds [34] with an excellent tolerance of the procedure. A first clinical trial of SVF injection which enrolled 8 patients was recently reported by our group [35] suggesting a clear improvement in the majority of the vocal parameters obtained at 12 months. However, the etiology of vocal fold scarring is very heterogenous so a better understanding of the mechanisms underlying the efficacy of ASC and SVF is mandatory. In addition, identification of in vitro potency assay able to anticipate the anti-fibrotic potential of the cell therapy product could allow a better selection of patients who can benefit from these innovative therapies.

Based on our results, it appears that adipose derived-cellular therapies are interesting candidates for reducing myofibroblast phenotype acquisition. Nevertheless, global approaches to further characterize ASC and SVF anti-fibrotic potential and studies on a large scale are further needed to better characterize the mechanisms of action.

\section{Abbreviations}


Vocal fold

ECM

extracellular matrix

aSMA

alpha-smooth-muscle actin

ACTA2

actin alpha 2

bFGF

basic Fibroblasts Growth Factor

HGF

Hepatocyte Growth Factor

MSC

Mesenchymal stromal cells

SVF

Stromal Vascular Fraction

ASC

adipose derived stromal cells

hVFF

human vocal fold fibroblasts

MMC

macromolecular crowding

TGF- $\beta 1$

transforming growth factor beta 1

DMEM

Dulbecco's modified Eagle's medium

FCS

fetal calf serum

SM

standard medium

GMP

Good Manufacturing Practices

RT

Reverse Transcription

RT-qPCR

Reverse Transcription quantitative Polymerase Chain Reaction GAPDH

glyceraldehyde-3-phosphate dehydrogenase

SDS-PAGE

sodium dodecylsulphate-polyacrylamide gel electrophoresis

VEGF 
vascular endothelial growth factor

COL1A1

Collagen 1 alpha 1

\section{Declarations}

\section{Ethics approval and consent to participate}

All patients provided informed consent for the scientific use of surgical residues.

Consent for publication: Not applicable

\section{Funding}

This work was supported by PHC Amadeus Campus 2017 (grant number FR05/2017).

\section{Availability of data and materials}

The datasets used and/or analysed during the current study are available from the corresponding author on reasonable request.

\section{Competing interests}

The authors declare that they have no competing interests.

\section{Author's contributions:}

Conceptualization : AM, AG, FS, MG, TG

Methology: MV, AM, JM, AG, FS, MG, TG

Funding acquisition: AM, AG, FS, MG, TG

Investigation: MV, AM, SS, LG, LA, TG

Data interpretation : MV, AM, SS, JM, AG, FDG, FS, MG, TG

Original draft : MV, AM

Revise the manuscript: JM, FDG, FS, MG, TG

All authors read and approved the final manuscript.

Acknowledgments: Not applicable

\section{References}


1. Hantzakos A, Dikkers FG, Giovanni A, Benninger MS, Remacle M, Sjögren EV, et al. Vocal fold scars: a common classification proposal by the American Laryngological Association and European Laryngological Society. Eur Arch Otorhinolaryngol. 2019 Aug;276(8):2289-92.

2. Friedrich G, Dikkers FG, Arens C, Remacle M, Hess M, Giovanni A, et al. Vocal fold scars: current concepts and future directions. Consensus report of the phonosurgery committee of the European laryngological society. Eur Arch Otorhinolaryngol. 2013 Sep;270(9):2491-507.

3. Wingstrand VL, Larsen CG, Jensen DH, Bork K, Sebbesen L, Balle J, et al. Mesenchymal Stem Cell Therapy for the Treatment of Vocal Fold Scarring: A Systematic Review of Preclinical Studies. PLOS ONE. 2016;12.

4. Kumai Y, Kobler JB, Park H, Galindo M, Herrera VLM, Zeitels SM. Modulation of vocal fold scar fibroblasts by adipose-derived stem/stromal cells. The Laryngoscope. 2009;n/a-n/a.

5. Graupp M, Bachna-Rotter S, Gerstenberger C, Friedrich G, Fröhlich-Sorger E, Kiesler K, et al. The unsolved chapter of vocal fold scars and how tissue engineering could help us solve the problem. Eur Arch Otorhinolaryngol. 2016 Sep;273(9):2279-84.

6. Sataloff RT, Spiegel JR, Heuer RJ, Baroody MM, Emerich KA, Hawkshaw MJ, et al. Laryngeal minimicroflap: A new technique and reassessment of the microflap saga. J Voice. 1995 Jun;9(2):198204.

7. Molteni G, Bergamini G, Ricci-Maccarini A, Marchese C, Ghidini A, Alicandri-Ciufelli M, et al. Autocrosslinked hyaluronan gel injections in phonosurgery. Otolaryngol Neck Surg. 2010 Apr;142(4):54753.

8. Gugatschka M, Ohno S, Saxena A, Hirano S. Regenerative medicine of the larynx. Where are we today? A review. J Voice Off J Voice Found. 2012 Sep;26(5):670.e7-13.

9. Suehiro A, Hirano S, Kishimoto Y, Tateya I, Rousseau B, Ito J. Effects of basic fibroblast growth factor on rat vocal fold fibroblasts. Ann Otol Rhinol Laryngol. 2010 Oct;119(10):690-6.

10. Suehiro A, Hirano S, Kishimoto Y, Rousseau B, Nakamura T, Ito J. Treatment of acute vocal fold scar with local injection of basic fibroblast growth factor: a canine study. Acta Otolaryngol (Stockh). 2010 Jul 1;130(7):844-50.

11. Kishimoto Y, Hirano S, Kitani Y, Suehiro A, Umeda H, Tateya I, et al. Chronic vocal fold scar restoration with hepatocyte growth factor hydrogel. The Laryngoscope. 2010 Jan;120(1):108-13.

12. Graupp M, Rinner B, Frisch MT, Weiss G, Fuchs J, Sundl M, et al. Towards an in vitro fibrogenesis model of human vocal fold scarring. Eur Arch Oto-Rhino-Laryngol Off J Eur Fed Oto-Rhino-Laryngol Soc EUFOS Affil Ger Soc Oto-Rhino-Laryngol - Head Neck Surg. 2018 May;275(5):1211-8.

13. Kishimoto Y, Hirano S, Suehiro A, Tateya I, Kanemaru S, Nakamura T, et al. Effect of exogenous hepatocyte growth factor on vocal fold fibroblasts. Ann Otol Rhinol Laryngol. 2009 Aug;118(8):60611.

14. Ohno T, French LC, Hirano S, Ossoff RH, Rousseau B. Effect of hepatocyte growth factor on gene expression of extracellular matrix during wound healing of the injured rat vocal fold. Ann Otol Rhinol Laryngol. 2008 Sep;117(9):696-702. 
15. Hirano S, Kawamoto A, Tateya I, Mizuta M, Kishimoto Y, Hiwatashi N, et al. A phase I/II exploratory clinical trial for intracordal injection of recombinant hepatocyte growth factor for vocal fold scar and sulcus. J Tissue Eng Regen Med. 2018 Apr;12(4):1031-8.

16. Friedenstein AJ, Gorskaja JF, Kulagina NN. Fibroblast precursors in normal and irradiated mouse hematopoietic organs. Exp Hematol. 1976 Sep;4(5):267-74.

17. Liang X, Ding Y, Zhang Y, Tse H-F, Lian Q. Paracrine Mechanisms of Mesenchymal Stem Cell-Based Therapy: Current Status and Perspectives. Cell Transplant. 2014 Sep;23(9):1045-59.

18. Spees JL, Lee RH, Gregory CA. Mechanisms of mesenchymal stem/stromal cell function. Stem Cell Res Ther. 2016 31;7(1):125.

19. Dykstra JA, Facile T, Patrick RJ, Francis KR, Milanovich S, Weimer JM, et al. Concise Review: Fat and Furious: Harnessing the Full Potential of Adipose-Derived Stromal Vascular Fraction. Stem Cells Transl Med. 2017;6(4):1096-108.

20. Graupp M, Gruber H-J, Weiss G, Kiesler K, Bachna-Rotter S, Friedrich G, et al. Establishing principles of macromolecular crowding for in vitro fibrosis research of the vocal fold lamina propria: MMC for Vocal Fold Fibrosis Research. The Laryngoscope. 2015 Jun;125(6):E203-9.

21. Chen X, Thibeault SL. Novel Isolation and Biochemical Characterization of Immortalized Fibroblasts for Tissue Engineering Vocal Fold Lamina Propria. Tissue Eng Part C Methods. 2009 Jun;15(2):20112.

22. Granel B, Daumas A, Jouve E, Harlé J-R, Nguyen P-S, Chabannon C, et al. Safety, tolerability and potential efficacy of injection of autologous adipose-derived stromal vascular fraction in the fingers of patients with systemic sclerosis: an open-label phase I trial. Ann Rheum Dis. 2015 Dec;74(12):2175-82.

23. Hata R-I, Senoo H. L-ascorbic acid 2-phosphate stimulates collagen accumulation, cell proliferation, and formation of a three-dimensional tissuelike substance by skin fibroblasts. J Cell Physiol. 1989 Jan;138(1):8-16.

24. Vyas B, Ishikawa K, Duflo S, Chen X, Thibeault SL. Inhibitory effects of hepatocyte growth factor and interleukin- 6 on transforming growth factor-beta1 mediated vocal fold fibroblast-myofibroblast differentiation. Ann Otol Rhinol Laryngol. 2010 May;119(5):350-7.

25. Chen C, Peng Y, Wang Z, Fish P, Kaar J, Koepsel R, et al. The Scar-in-a-Jar: studying potential antifibrotic compounds from the epigenetic to extracellular level in a single well: Scar-in-a-Jar to assess potential antifibrotics. Br J Pharmacol. 2009 Nov;158(5):1196-209.

26. Chen C, Loe F, Blocki A, Peng Y, Raghunath M. Applying macromolecular crowding to enhance extracellular matrix deposition and its remodeling in vitro for tissue engineering and cell-based therapies. Adv Drug Deliv Rev. 2011 Apr;63(4-5):277-90.

27. Karbiener M, Darnhofer B, Frisch M-T, Rinner B, Birner-Gruenberger R, Gugatschka M. Comparative proteomics of paired vocal fold and oral mucosa fibroblasts. J Proteomics. 2017 Feb;155:11-21.

28. Livak KJ, Schmittgen TD. Analysis of Relative Gene Expression Data Using Real-Time Quantitative PCR and the 2- $\triangle \Delta C T$ Method. Methods. 2001 Dec;25(4):402-8. 
29. Steinmann B, Rao VH, Vogel A, Bruckner P, Gitzelmann R, Byers PH. Cysteine in the triple-helical domain of one allelic product of the alpha $1(I)$ gene of type I collagen produces a lethal form of osteogenesis imperfecta. J Biol Chem. 1984 Sep 10;259(17):11129-38.

30. Bronckaers A, Hilkens P, Martens W, Gervois P, Ratajczak J, Struys T, et al. Mesenchymal stem/stromal cells as a pharmacological and therapeutic approach to accelerate angiogenesis. Pharmacol Ther. 2014 Aug;143(2):181-96.

31. Nauta AJ, Fibbe WE. Immunomodulatory properties of mesenchymal stromal cells. Blood. 2007 Nov 15;110(10):3499-506.

32. Yasuda K, Ozaki T, Saka Y, Yamamoto T, Gotoh M, Ito Y, et al. Autologous cell therapy for cisplatininduced acute kidney injury by using non-expanded adipose tissue-derived cells. Cytotherapy. 2012 Oct;14(9):1089-100.

33. Hirose Y, Funahashi Y, Matsukawa Y, Majima T, Yamaguchi M, Kawabata S, et al. Comparison of trophic factors secreted from human adipose-derived stromal vascular fraction with those from adipose-derived stromal/stem cells in the same individuals. Cytotherapy. 2018;20(4):589-91.

34. Mattei A, Magalon J, Bertrand B, Philandrianos C, Veran J, Giovanni A. Cell therapy and vocal fold scarring. Eur Ann Otorhinolaryngol Head Neck Dis. 2017 Oct 1;134(5):339-45.

35. Mattei A, Bertrand B, Jouve E, Blaise T, Philandrianos C, Grimaud F, et al. Feasibility of First Injection of Autologous Adipose Tissue-Derived Stromal Vascular Fraction in Human Scarred Vocal Folds: A Nonrandomized Controlled Trial. JAMA Otolaryngol Neck Surg [Internet]. 2020 Feb 13; Available from: ttps://jamanetwork.com/journals/jamaotolaryngology/fullarticle/2760908

\section{Table}

Table 1: Primer sequences used for RT-qPCR

\begin{tabular}{ccccc}
\hline Gene & Gene symbol & Forward primer & Reverse primer & Product lenght [bp] \\
\hline $\begin{array}{c}\text { Alpha smooth } \\
\text { muscle actin }\end{array}$ & ACTA2 & CGTTACTACTGCTGAGCGTGA & GCCCATCAGGCAACTCGTAA & 137 \\
$\begin{array}{c}\text { Beta-2- } \\
\text { microglobulin }\end{array}$ & B2M & AGGCTATCCAGCGTACTCCA & CGGATGGATGAAACCCAGACA & 105 \\
Collagen I $\boldsymbol{\alpha} \mathbf{1}$ & COL1A1 & CCCCGAGGCTCTGAAGGT & GCAATACCAGGAGCACCATTG & 140 \\
\hline
\end{tabular}

\section{Figures}


A

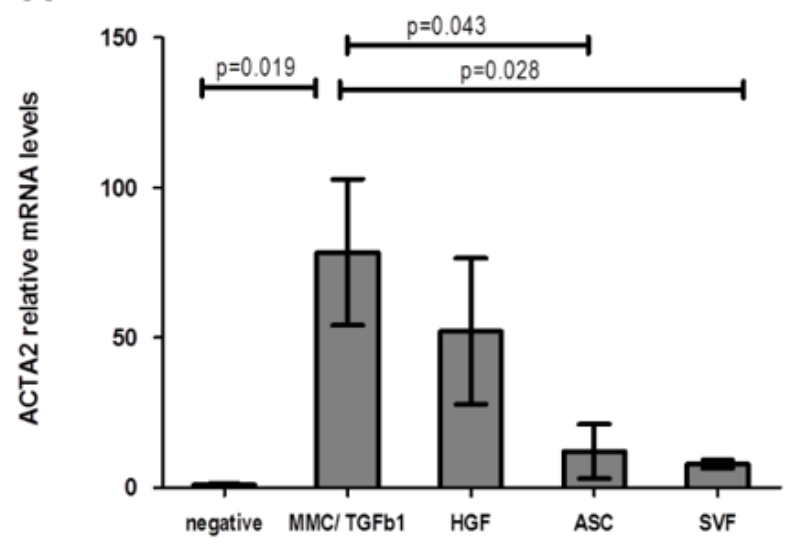

B
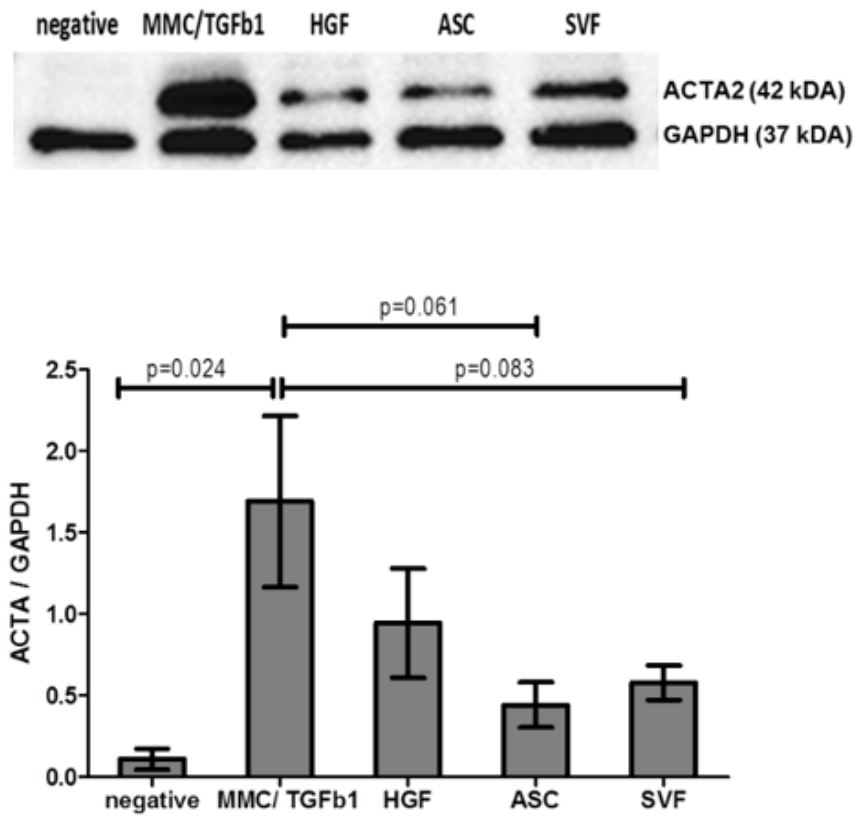

\section{Figure 1}

(A) mRNA expression level of ACTA2 by RT-qPCR. Data is presented as mean \pm S.E.M.. Statistical analysis was performed using Mann-Whitney test. (B) The protein level of ACTA2 was examined by Western blot analysis on whole cells lysates from hVFF after co-culture with ASC $(n=4)$ and SVF $(n=4)$. Representative blots for ACTA2 and GAPDH (loading control) are shown. (C) Densitometric data from ACTA2 protein levels. Data is presented as mean \pm S.E.M., normalized to GAPDH protein levels and relative to the untreated condition (negative). Statistical analysis was performed using Mann-Whitney test

A

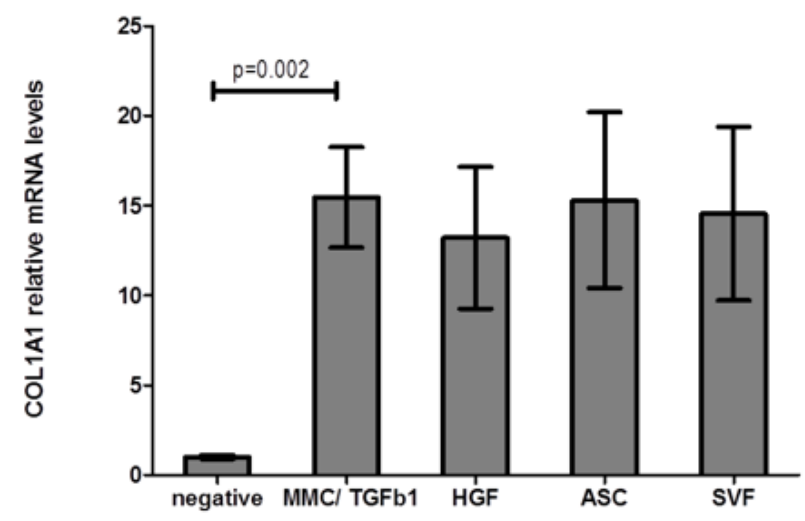

B

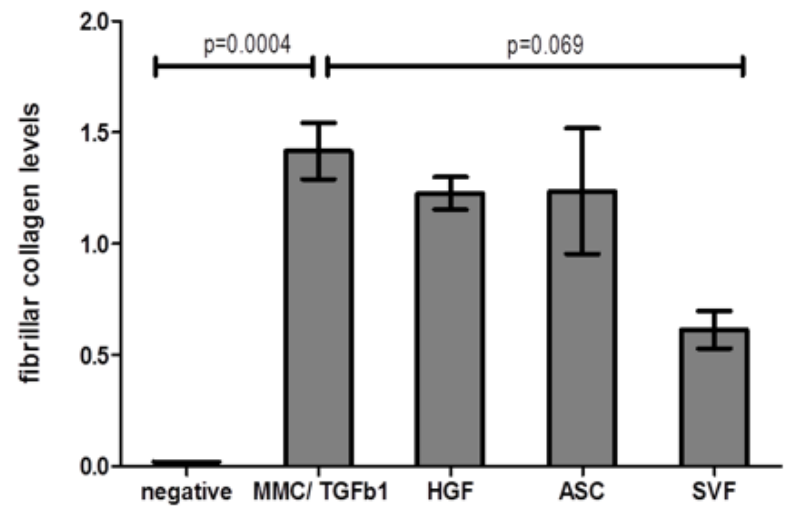

Figure 2

(A) mRNA expression level of COL1A1 by RT-qPCR. Data is presented as mean \pm S.E.M.. Statistical analysis was performed using Mann-Whitney test. (B) Densitometric data from fibrillar collagen I protein levels. Data is presented as mean \pm S.E.M., normalized to collagen I protein levels of alpha2 chain under 
crowded condition with TGF- $\beta 1$, and relative to the untreated condition (negative). Statistical analysis was performed using Mann-Whitney test 